ranga. The database can be updated and edited. The protein spectra files can be entered into "MALDI Biotyper 3.0" and newer versions database by creating reference spectra and importing them into the Taxonomic Tree.

Protein spectra were obtained at 10-20-fold study of the samples extracted with acetonitrile/formic acid on a mass spectrometer "Microflex LT" (Bruker Daltonics, Germany) using a "Flex Control 3.3" program. The database was tested with eight strains isolated in Siberia and at the Far East in 2012-2016, and 18 Leptospira cultures from the collection of Gamaleya State Research Centre for Epidemiology and Microbiology. The results of identification of pathogenic Leptospira completely coincided with the data of multilocus sequencing. The created database is intended for specialists of microbiological and scientific laboratories engaged in diagnostics and study of leptospirosis.

\section{INFORMATION TECHNOLOGY APPLICATION FOR NATURAL FOCI INFECTIONS MONITORING AND PREDICTION}

\section{A.V. Burdakov, A.O. Ukharov}

Moscow State Technical University n.a. N.E.Bauman, Moscow, Russia

Amur Region's climate-geographical features, flora and fauna specifics led to the emergence of persistent natural foci of several infections, including tick-borne encephalitis, hemorrhagic fever, tularemia, and listeriosis. Amur Region registers from 100 to 200 cases of natural foci infections every year. The largest share of these infections is tick-borne.

The Amur Region Rospotrebnadzor Service introduced in 2017 the Epidemiological Surveillance System (EpiS), creating one information space for the entire epidemiological service network in the region and ensuring connectivity with the primary healthcare organizations. EpiS enabled rapid collection and exchange of information, supports epidemiological investigations with accurate geo-location based on GLONASS/GPS coordinates and delivers outbreak early warning capabilities. The natural foci locations and Anthrax cases historical data for the past 180 years was converted into an electronic register of the territories and sites and embedded into the EpiS. It is planned to expand this registry for other natural foci infections.

Operational information on morbidity in conjunction with the historical information and other epidemiologically significant factors is displayed in the Emergency Operations Center (EOC) in near real-time on detailed regional maps. The EOC strengthens and supports current situation monitoring as well as in-depth epidemiological investigation and analysis of the situation.

In 2018 the Amur Region Rospotrebnadzor Service also introduced modern methods for predicting morbidity based on the deep neural networks technology. This forecasting method demonstrated its effectiveness for a number of infections, in particular, Influenza-like illness (ILI) and Acute Intestinal infections, due to the integrated consideration of historical data, socio-hygienic characteristics and environmental factors. The prognostic models are built on the entire available data archive and allow identifying stable patterns leading to a change in the morbidity dynamics. The prediction accuracy reached $85-90 \%$ for the ILI. The use of such forecasting methods allows strengthening preventive measures to combat infectious morbidity. doi: 10.15789/2220-7619-2018-4-4.7

\section{ANALYSIS AND FORECASTING INCIDENCE} OF BRUCELLOSIS IN THE REPUBLIC OF DAGESTAN

A.A. Dalgatova ${ }^{1}$, L.V. Sayapina ${ }^{2}$, V.B. Postupailo ${ }^{2}$, O.A. Burgasova ${ }^{3}$, N.F. Nikityuk ${ }^{2}$

${ }^{1}$ SBN of the Republic of Dagestan "Charodinskaya Central District Hospital" of the Russia, ${ }^{2}$ Scientific Center for Examination of Means of Medical Application, Moscow, Russia; ${ }^{3}$ Peoples' Friendship

University of Russia (RUDN University), Moscow, Russia

From 300 to 500 cases of brucellosis among people are registered in Russian Federation annually. Sixty three percent of these cases occur in the North Caucasus Federal District (NCFD). Assessing the structure of the brucellosis incidences among the population of NCFD during 2005-2017 we found that 2122 reported cases (67\%) occurred in the Republic of Dagestan.

The current situation calls for the need to improve methods of epidemiological diagnosis. To assess the epidemiological situation of brucellosis and to forecast the epidemic situation in the Republic of Dagestan for 2018 we analyzed the official statistics of brucellosis cases among people. The forecast for the number of cases was executed using two methods - the classical method of linear approximation and by the method developed by us, using the Wald's graph plot. The proposed method allows to determine the monthly minimum and maximum number of cases of brucellosis in the forthcoming period, therefore predicting the total minimum and maximum levels of morbidity.

According to the result of the statistical analysis, the threshold level of incidence of brucellosis disease (Mediterranean fever) in the Republic of Dagestan between 2005-2017 amounted to 14 cases. The average values of incidence of disease during long-term observations fluctuated between 12 and 20 cases during different seasons of the year, with the most cases occurring in June.

As a result of the conducted analysis, it was established that in the Republic of Dagestan in 2018, the monthly increase in the incidence level amounted to 1.6 new cases of brucellosis, the aggregate minimum prognosis of the incidence level is 10 new cases, while the maximum prognosis is 28 new cases. 123 cases are forecast in the Republic of Dagestan in 2018.

Therefore, according to the latest findings utilizing Wald's graph plot, it has been established that the brucellosis epidemiological situation in the Republic of Dagestan in 2018 is unstable. The exceedance of the threshold level points to the possible decline of the epidemiological situation. The suggested method of brucellosis epidemiological case forecast contributes to the optimization of the epidemiological process management, allows for the timely evaluation of the activity of the epidemic outbreak areas and prompt and swift decision making for the purposes of their localization and liquidation.

\section{8} doi: 10.15789/2220-7619-2018-4-4.8

\section{THE DYNAMICS OF TICK VECTORS INFECTION RATE WITH IXODIC TICK BORRELIOSIS CAUSATIVE AGENTS IN KHABAROVSK REGION DURING THE EPIDEMIC} SEASON OF 2017-2018

\section{A.G. Dragomeretskaya, A.P. Romanova, O.E. Trotsenko,} T.V. Mzhelskaya

Khabarovsk research institute of epidemiology and microbiology, Khabarovsk, Russia

Ixodic tick borreliosis are among the most prevalent illnesses in the group of tick-borne diseases in Russia.

The objective of the research was to perform a comparative evaluation of infestation rate of engorged Ixodic 
ticks with Borrelia burgdorferi sensu lato (B. burgdorferi s.1.) and Borrelia miyamotoi in the Khabarovsk region during 2017-2018.

A total number of 1238 ixodic ticks were tested on the presence of the $B$. burgdorferi s.l. and 710 ticks were tested on the presence of B. miyamotoi DNA via Real-time PCR. Identification of the nucleic acids of the pathogens was performed using PCR kits "RealBest DNA B. miyamotoi", "RealBest DNA B. burgdorferi s.l." ("Vector-Best", Novosibirsk) according to the manufacturer's instructions.

The infestation rate of Ixodes persulcatus ticks $(31.4 \pm 1.74 \%)$ with $B$. burgdorferi s.l. was statistically higher compared to Dermacentor silvarum $(12.8 \pm 4.87 \%, \mathrm{p}<0.05)$ and Haemaphysalis spp. $(16.6 \pm 2.86 \%, \mathrm{p}<0.05)$. No significant difference between infestation rates of different species of ticks with B. miyamotoi was found. During the start of the epidemic season was registered an elevation of vectors infestation rate with $B$. burgdorferi s.l. with a peak in July $(33.1 \pm 4.33 \%, \mathrm{p}<0.05)$ followed by a consequent decline down to $11.8 \pm 5.53 \%$ ( $p<0.05$ ) in September. A decline in infestation rate of $B$. miyamotoi vectors from $10.4 \pm 2.63$ to $5.8 \pm 1.46 \%(\mathrm{p}<0.05)$ was registered. From July to September the DNA of B. miyamotoi was not found. It is of importance that 14 ticks had a coinfection with B. burgdorferi and B. miyamotoi in 2017-2018. The Ct DNA value of $B$. burgdorferi s.l. was higher in most of the cases compared with Ct DNA value of B. miyamotoi.

The infestation rate of Ixodic ticks with $B$. miyamoto $i$ was significantly lower compared to $B$. burgdorferi s.l. in Khabarovsk Region. The obtained results imply that during the start of the epidemic season (April-May) the risk of exposure of the population to $B$. miyamoto is higher compared to summer-autumn period. Thus, it is important to study the competition between the pathogens in ticks and its value on the manifestation of the diseases in humans.

\section{PATHOGENS, PESTICIDE RESISTANCE AND GENETIC} DIVERSITY OF HUMAN HEAD LICE

M.E. Eremeeva ${ }^{1}$, M. Anderson ${ }^{1}$, T. Ogunleke ${ }^{1}$, S. Warang ${ }^{1}$, D. Capps ${ }^{1}$, S. Zohdy ${ }^{2}$, L.A. Durden ${ }^{3}$

'Jiann-Ping Hsu College of Public Health, Georgia Southern University, Statesboro, USA; ${ }^{2}$ School of Forestry \& Wildlife Sciences, Auburn University, Auburn, USA; ${ }^{3}$ College of Science and Mathematics, Georgia Southern University, Statesboro, USA

Pediculosis capitis or head louse infestation is the most prevalent parasitic infestation of humans. It is commonly perceived as an age-dependent rite of passage, and as an embarrassing social nuisance; however, head louse outbreaks do not raise any substantial public health concerns due to their assumed low capacity for transmitting the louse-borne pathogens associated with Pediculus humanus humanus, the human body louse. The purpose of this study was to screen head lice, Pediculus humanus capitis from Georgia, USA and Madagascar for Bartonella quintana and Acinetobacter sp. to determine the risk of exposure of rural populations with different levels of economic development to these pathogens. Other aims were to examine these lice for the occurrence of genetic markers for permethrin resistance using restriction fragment length polymorphism (RFLP/PCR) analysis and to evaluate the genetic structure of these head louse populations using microsatellite typing. The $k d r$ permethrin resistance biomarker for the T917I mutation was detected by RFLP/PCR in 99.9 and $70 \%$ of lice from Georgia and Madagascar, respectively.
Bartonella DNA was detected at similar levels in both set of samples (10.3 and 12.6\%), while Acinetobacter sp. DNA was detected more frequently in Georgia lice $(80.8 \%)$ than in Malagasy lice $(42.1 \%)$. Microsatellite typing based on 3 sites revealed significant genetic heterogeneity among the lice tested, although head lice from Georgia were separated in 2 closely related clusters, while Malagasy lice exhibited more genetic diversity using Principal Component Analysis and Bayesian clustering. The results provide the first information regarding these combined characteristics of head louse infestations at these locations and can be used as a baseline for temporal surveillance of changes in circulating head louse populations, for monitoring louse susceptibility to permethrin-based pediculicides, and to track potential exposures and outbreaks due to louse-borne pathogens.

\section{WHOLE GENOME-BASED CHARACTERIZATION OF COXIELLA BURNETII STRAINS ISOLATED IN RUSSIAN FEDERATION}

O.A. Freylikhman ${ }^{1,2}$, A.M. Kiselev' ${ }^{2}$, A.A. Sergushichev ${ }^{3}$, S.V. Kazakov ${ }^{3}$, Yu.A. Panferova ${ }^{1}$, A.A. Kostareva ${ }^{2,3}$, N.K. Tokarevich ${ }^{1}$

${ }^{1}$ St. Petersburg Pasteur Institute, St. Petersburg, Russia $;{ }^{2}$ Federal Almazov North-West Medical Research Centre, St. Petersburg, Russia $;{ }^{3}$ ITMO University, St. Petersburg, Russia

Information on the whole genome structure of the Coxiella burnetii strains circulating in Russian Federation (RF) is currently unavailable. The identification and study of differential-significant genetic markers of C. burnetii that are important from the epidemiological point of view remains a priority, including determining the hostal specificity.

The aim was whole genome-based characterization of $C$. burnetii strains isolated from different hosts.

We performed whole genome sequencing of four C. burnetii strains isolated from two host types (the human and the arthropods) in RF using the MiSeq technology (Illumina, USA). Genome assembling and alignement using Dugway 5J108-111 as the reference was performed with SPADes 3.9.0 genome assembler. Comparative analysis of the whole genomes of Russian strains was carried out between the investigated genomes and the whole genomes sequences of $C$. burnetii available in the NCBI database.

It was confirmed the conception of closed pangenome of species $C$. burnetii characterized by a low intraspecies genetic diversity, including the population circulating in the territory of RF. However, in Russian strains of $C$. burneti unlike foreign strains the analysis of the variable part of the genome and the composition of unique genes revealed deletions of a part of them, which allows us to speak about their unique genomotype. Analysis showed pronounced clustering within a group of Russian strains by host type, the differences between genomes within clusters were minor. Comparing the number of deleted genome fragments it was found that surprisingly strains from arthropods had a significantly greater genome reduction compared with strains from human. These data are in contrast with the conclusions of a number of authors that the genomic reduction of $C$. burnetii strains isolated from arthropods is limited.

Thus, this corpus of data allow us to characterize the genomes of $C$. burnetii strains isolated in the territory of RF and to make assumptions about the hostal specificity of this pathogen, prompting further studies of its mechanisms. 